\title{
Fracture mechanics model of stone comminution in ESWL and implications for tissue damage
}

\author{
Murtuza Lokhandwalla and Bradford Sturtevant $\dagger$ \\ Graduate Aeronautical Laboratories, California Institute of Technology, \\ Pasadena, California, USA \\ E-mail: brad@galcit.caltech.edu
}

Received 20 January 2000

\begin{abstract}
Focused shock waves administered during extracorporeal shock-wave lithotripsy (ESWL) cause stone fragmentation. The process of stone fragmentation is described in terms of a dynamic fracture process. As is characteristic of all brittle materials, fragmentation requires nucleation, growth and coalescence of flaws, caused by a tensile or shear stress. The mechanisms, operative in the stone, inducing these stresses have been identified as spall and compressioninduced tensile microcracks, nucleating at pre-existing flaws. These mechanisms are driven by the lithotripter-generated shock wave and possibly also by cavitation effects in the surrounding fluid. In this paper, the spall mechanism has been analysed, using a cohesive-zone model for the material. The influence of shock wave parameters, and physical properties of stone, on stone comminution is described. The analysis suggests a potential means to exploit the difference between the stone and tissue physical properties, so as to make stone comminution more effective, without increasing tissue damage.
\end{abstract}

\section{Introduction}

Fragmentation of kidney stones using focused shock waves forms the basis of extracorporeal shock-wave lithotripsy (ESWL). Several thousand shock waves are administered during this treatment so as to attain a desirable size distribution of the fragmented stone, which could then be discharged naturally. Based on usage statistics worldwide (Chaussy and Fuchs 1989), this treatment has been generally considered effective for stone removal in a majority of cases, eliminating the need for surgery.

Despite its success, the mechanism of stone fragmentation and concomitant tissue injury (Kaude et al 1985), due to focused waves, needs to be understood. Significant progress has been made in the past, characterizing kidney stones on the basis of their mechanical properties, stone composition, shape and size. Attempts have been made to correlate stone attributes, properties of the surrounding fluid and waveform parameters, with susceptibility of material to fragment. Several mechanisms for stone fragmentation have been proposed and documented in the literature. A list of references to works which examine the above issues is provided in a review paper by Coleman and Saunders (1993).

Figure 1 summarizes stone fracture mechanisms. A distinction is made between the processes operative in the fluid surrounding the stone, which cause stone fragmentation, and

$\dagger$ Author to whom correspondence should be addressed at: Caltech 301-46, Pasadena, CA 91125, USA. See: http://www.galcit.caltech.edu/ brad 


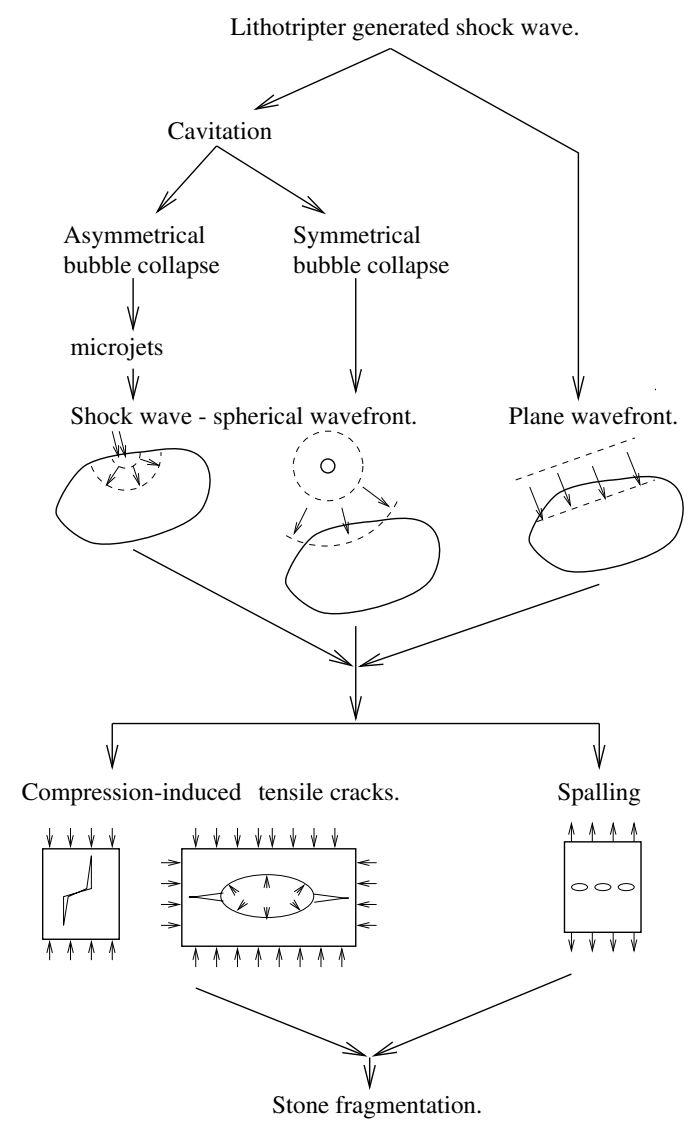

Figure 1. Mechanisms of stone fracture.

the mechanics of stone fragmentation itself. The mechanics which we are referring to in this paper are interior to the stone. A typical pressure pulse generated by an electro-hydraulic shock wave lithotripter is shown in figure 2. It involves a steep compressive front with pressures of the order of $40 \mathrm{MPa}$, decaying to a negative pressure of $10 \mathrm{MPa}$, and the entire pulse lasting for a duration of $4 \mu \mathrm{s}$. Pressure measurements (Robin et al 2000, Müller 1990) near the focal region of a Dornier HM3 lithotripter, indicate a $6 \mathrm{~dB}$ beam width of approximately $15 \mathrm{~mm}$. Since kidney stones are also, typically, of the same dimension, the wave front incident on the stone can be considered as a plane wave. Also, experiments on weak wave focusing (Sturtevant and Kulkarny 1976) indicate the existence of a Mach stem in the focal region, implying that the wave front geometry is nearly plane, locally. This plane wave propagates through the stone, first exerting a compressive stress and then a tensile stress (figure 2).

The lithotripter-generated pressure field has been found to induce cavitation (Coleman et al 1987) in in vitro experiments. Negative pressure in the trailing part of the pulse causes bubbles to grow at nucleation sites. Ensuing pulses cause these bubbles to collapse. Bubble collapse adjacent to a solid surface is asymmetric, leading to a small jet of liquid impinging on the solid surface. Measured jet velocities are of the order of $100 \mathrm{~m} \mathrm{~s}^{-1}$ (Blake and Gibson 1987). Jet velocities and diameter depend on bubble diameter at collapse. The impinging jet sets up a locally compressive stress field in the stone, propagating spherically into the stone interior. 


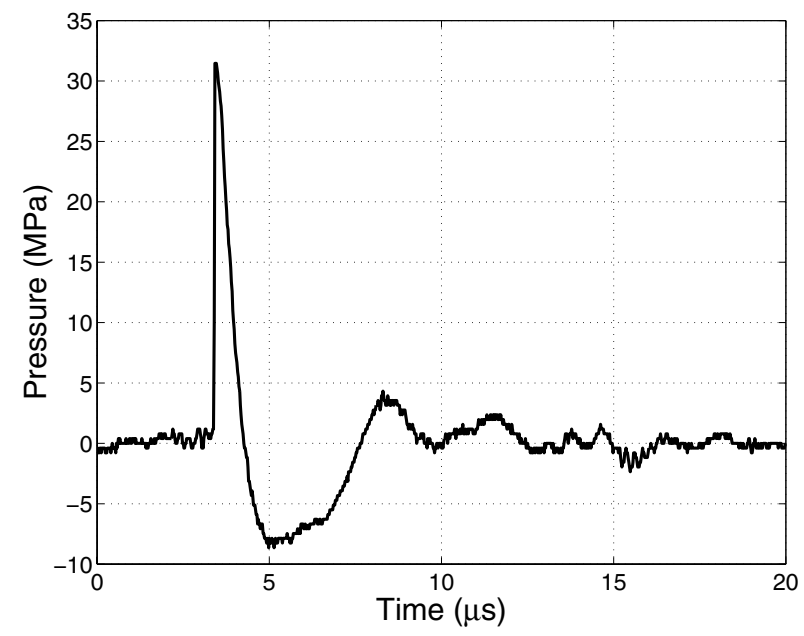

Figure 2. Pressure pulse at the lithotripter focus.

The stress fields generated by cavitation and the lithotripter pressure field are both potential candidates for driving fracture mechanisms in the interior of the stone, as indicated in figure 1. We propose to classify the stone fracture mechanisms as either a spall mechanism or failure due to compression-induced tensile cracks. Spalling refers to failure due to tensile stress, appearing as a result of reflection of the compressive part of the pulse at locations in the stone having an impedance mismatch. One such location is obviously the distal surface of the stone, but there could be other internal sites, for example cavities in the stone and the interface of crystalline and matrix materials. These tensile stresses cause nucleation and growth of microcracks, which eventually coalesce resulting in stone fragmentation. The failure plane, in this case, is perpendicular to the applied tensile stress. The trailing negative pressure of the lithotripter pulse also exerts tensile stresses, of a similar order of magnitude as in the spall mechanism. The resulting failure may not be called as a spall failure, but the model and analysis remain identical.

Contrary to the spall mechanism, where the stone is subjected to an overall tensile stress, an overall compressive loading sets up a local tensile stress field in the neighbourhood of preexisting defects such as grain boundaries, cavities, inclusions and similar flaws. These local tensile stresses, for brittle materials like stones and ceramics, would again result in microcrack nucleation and failure, as in the spall case. In this case the failure plane is axial, i.e. parallel to the direction of the applied compressive stress. This kind of failure is fairly common in rocks and has been well documented in the fracture mechanics literature (e.g. Brace and Bombolakis 1963, Bombolakis 1968).

To analyse spall failure, a cohesive zone model (Ortiz 1988) will be used in this work, with suitable modifications from its original form. This model is essentially a constitutive law for the material accounting for the presence of microcracks. The results from this analysis relate the physical properties of the stone, i.e. fracture toughness, acoustic speed, density and void dimensions, to the shock wave parameters, i.e. peak pressure, pulse width, pulse profile and number of shocks for fragmentation. In the next section a brief introduction to some basic fracture concepts is given, followed by a description of the cohesive zone model and an analysis of spall failure with this model. The number of shocks for spall failure are calculated using typical shock wave parameters and stone properties. Similar calculations can also be 
performed for the compression failure mechanism (Nemat-Nasser and Deng 1994), which will be dealt with in future work.

An essential feature of SWL is that the damage inflicted by the lithotripter shock waves accumulates during the course of the treatment, leading to eventual destruction of stone and the tissue material. The model used in this work enables us to determine this progressive damage, with each passing shock wave. In this work we define damage parameters for stone and tissue material. The term damage is applied to an irreversible impairment of mechanical properties of a material due to unfavourable mechanical and environmental conditions. Some of the prominent damage modes are enlisted below:

(a) Thermomechanical damage (creep): occurs at high temperatures under action of stress.

(b) Chemomechanical damage (stress corrosion): materials subjected to stress, immersed in an aggressive media.

(c) Fatigue damage: growth and coalescence of microcracks, due to action of cyclic stress.

Damage models are described in the literature (e.g. Kachanov 1986) for each of the above modes. For SWL, we are concerned primarily with fatigue damage. In earlier work (Howard and Sturtevant 1997), a failure dose was defined for nitrocellulose membranes using the principle of linear summation of damage (Miner 1945). It can be shown that the damage model used here gives results consistent with this principle. A natural outcome of this analysis is that it provides us with a common ground for comparing the damage done to tissue and stone material, as the treatment progresses, due to various mechanisms. A section is devoted in this paper to providing a theoretical framework to exploit the differential response of the tissue and stone, and modify the shock wave parameters, to minimize tissue damage and improve stone comminution.

\section{Fracture mechanics concepts}

\subsection{Brittle fracture}

Engineering materials are usually characterized by their stress-strain $(\sigma-\epsilon)$ behaviour, typically obtained from a uniaxial loading configuration. Figure 3 shows the two most commonly observed stress-strain behaviours, i.e. $(a)$ ductile and $(b)$ brittle failure of materials. From the above behaviour, brittle fracture is defined as one in which the solid absorbs only a small amount of energy prior to failure, in contrast to a ductile fracture which involves extensive plastic deformation. It should be noted that materials are not inherently ductile or plastic, but it is a combination of their intrinsic properties (e.g. crystal structure, presence of flaws, impurities, etc) and extrinsic loading conditions (e.g. strain rate $(\dot{\epsilon})$, temperature) which lends to this gross behaviour. As indicated in figure 4, a material which has extensive plastic deformation at low strain rates tends to fail in a brittle manner at high strain rates. The stressstrain behaviour of typical kidney stone materials indicate very little to no plastic deformation, under a quasi-steady $\left(\dot{\epsilon} \sim 10^{-3} \mathrm{~s}^{-1}\right)$ uniaxial test (Ebrahimi and Wang 1989). Thus, when subjected to the lithotripter conditions of high strain rates $\left(\sim 10^{3} \mathrm{~s}^{-1}\right)$ the stones invariably exhibit a brittle fracture mode.

Griffith (1920-1) postulated that brittle fracture was due to the presence of small cracks in solids. According to his theory, at sufficiently high stresses these cracks propagate through the material, resulting in failure. For most crystalline materials there is competition between the material's tendency to deform plastically, with attendant work-hardening, and the tendency of the flaws to propagate and fragment the material. The amount of plastic deformation borne by a material is always finite, unless these flaws are eliminated, for example by heat treatment 

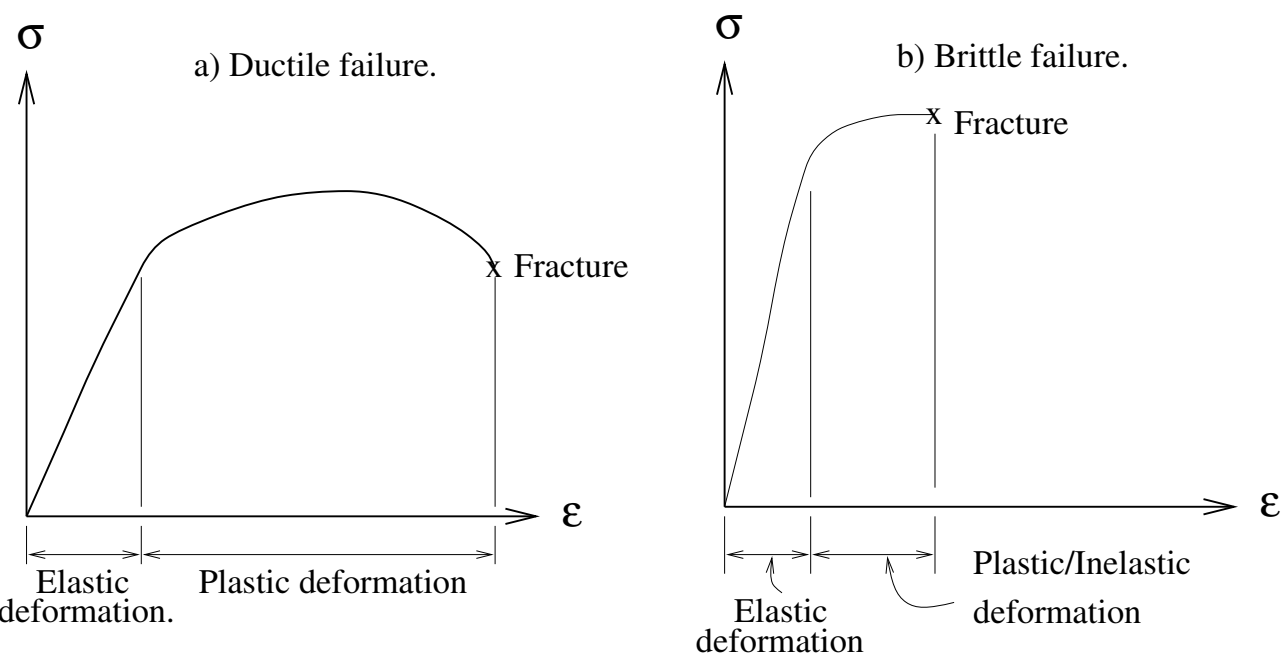

Figure 3. Stress-strain behaviour: (a) ductile failure, $(b)$ brittle failure.

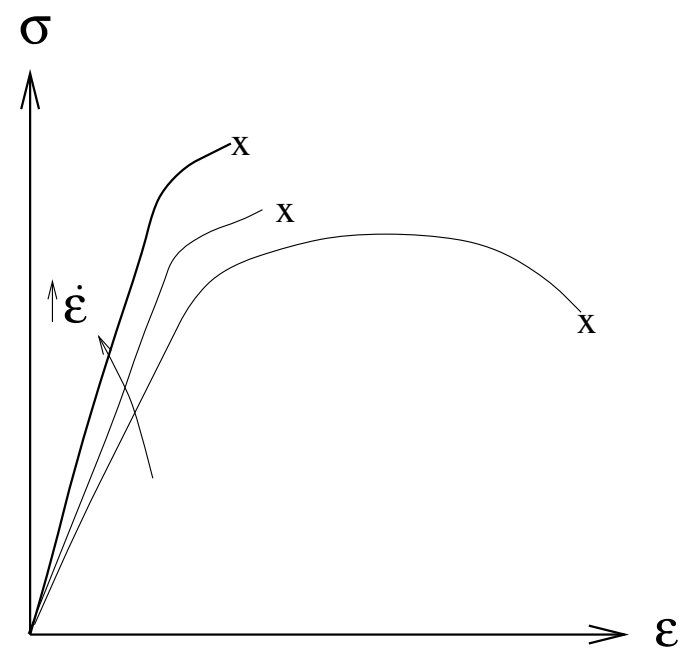

Figure 4. Strain-rate $(\dot{\epsilon})$ dependence.

in the case of metals. Thus the tendency to fracture always wins over. Figure 5 shows a thin, planar, sharp-edged crack. The stress field close to the crack tip can be obtained using the assumptions of a linear, unbounded, homogeneous, elastic solid, and is given as:

$$
\sigma_{i j}(r, \theta)=\frac{K_{I}}{\sqrt{2 \pi r}} f_{i j}(\theta)
$$

where $i, j=1,2,(r, \theta)$ are the polar coordinates of a point with the crack tip as the origin, $f_{i j}$ is a trigonometric function of $\theta$, and $K_{I}$ is the stress-intensity factor dependent on geometry and load configuration. Thus, according to (1), all the stresses tend toward infinity as we approach the crack tip. This never occurs in reality because of plastic deformation of the material near the crack tip. The stress-intensity factor is the single most important parameter describing the stress field near the crack. The parameter $K_{I}^{2} / E$, denoted as $G_{I}$ represents the energy 


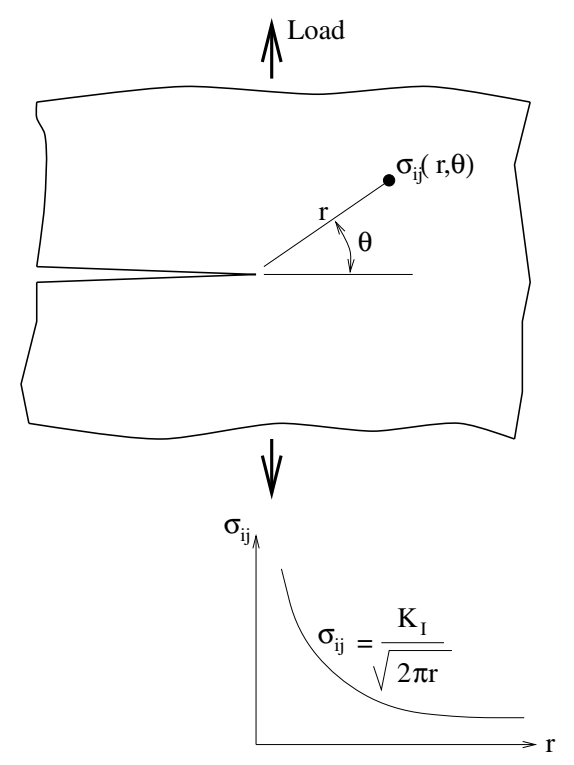

Figure 5. Stress field at the crack tip.

released by the crack per unit length of crack extension, where $E$ is the modulus of elasticity. As the load is increased, $K_{I}$, and consequently $G_{I}$, also increase, and at some point reach a critical value, causing the crack to grow. This critical value of stress-intensity factor, denoted as $K_{I C}$, is known as fracture toughness of the material. At criticality the strain energy lost by the cracked solid just balances the energy gained due to formation of new surfaces. Thus, the postulated fracture criterion is:

$$
K_{I}(\text { load, geometry })=K_{I C}(\text { material }) .
$$

Fracture toughness is an inherent material property and needs to be experimentally determined. It is one of the important indicators of the suitability of a particular kind of stone for ESWL treatment. For most metals and alloys, fracture toughness ranges between 20 and $200 \mathrm{MPa} \mathrm{m}^{1 / 2}$, whereas for rocks, ceramics and glass it ranges between 1 and $10 \mathrm{MPa} \mathrm{m}^{1 / 2}$. Values for renal calculi have not yet been measured, but are likely to lie in the latter range.

\subsection{Cohesive-zone model}

The criterion postulated above is true for initiation of a single crack in an otherwise homogeneous elastic solid. However, a typical scenario for stone fragmentation is as sketched in figure 6, wherein extensive microcracking has occurred within a region surrounding the crack tip. These microcracks would be distributed all around the main crack, but to make analysis tractable they are assumed to occur in the crack plane. The presence of a microcracked region facilitates crack growth by coalescence with microcracks. The effect of this microcracking on the main crack is taken into account by introducing a cohesive zone (Ortiz 1988). The cohesive zone model was originally proposed by Dugdale (1960) and Barenblatt (1962), and has since been used in the field of fracture mechanics (e.g. Achenbach et al 1979, Needleman 1987) for solving various problems.

Referring to figure 6 , the undamaged material has microcracks of length $a_{0}$, spaced at a distance $l$. Renal calculi have either a lamellar crystalline structure bonded by an organic 


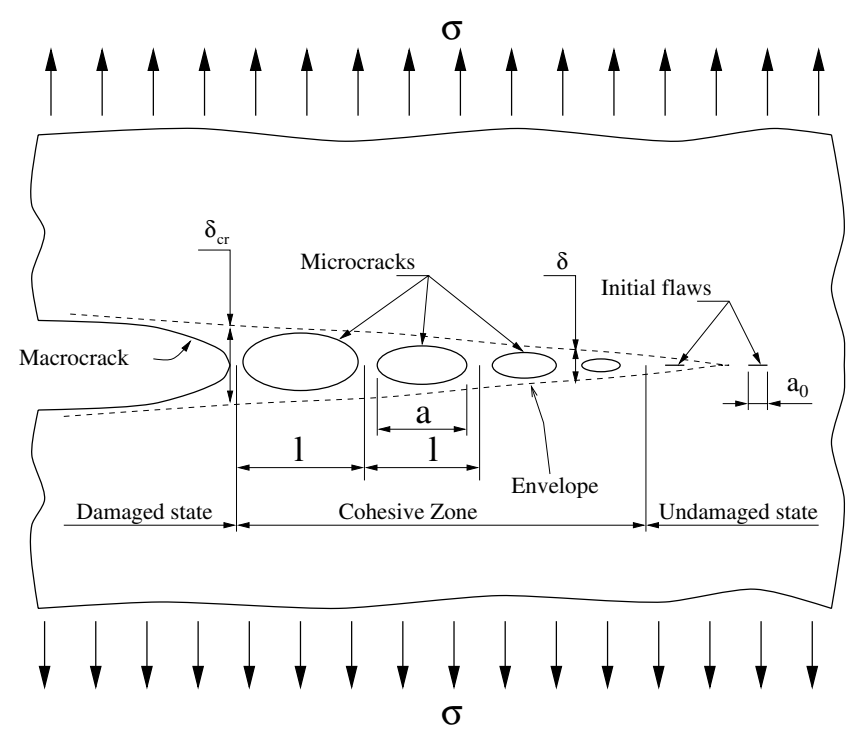

Figure 6. Coalescence of microcracks with main crack, and cohesive zone.

matrix material or an agglomerate of crystalline and non-crystalline material. The crack studied here is typically an interlamellar crack for the former case, or an intergranular crack for the agglomerate case, and the microcracks are flaws at the crystal boundaries. Hence the length scale, $l$, is of the order of grain size. When stressed, the microcracks would grow from their initial dimension $a_{0}$, to some dimension $a,\left(a_{0}<a<l\right)$, until eventually $a=l$, the microcrack has coalesced with the main crack. Or in terms of the opening displacement of the cohesive zone, $\delta$, when undamaged $\delta=0$, and coalescence occurs for some $\delta=\delta_{\mathrm{cr}}$. This critical opening displacement, $\delta_{\mathrm{cr}}$, is given in terms of material properties (Ortiz 1988):

$$
\frac{\delta_{\mathrm{cr}}}{l} \approx \frac{8}{\pi} \frac{1-v^{2}}{E} \frac{K_{I C}}{\sqrt{\pi l}} .
$$

A stress-displacement $(\sigma-\delta)$ relation for the cohesive zone, in accordance with the fracture criterion (2), is derived by Ortiz (1988). However it suffices for us to consider an approximate form of this relationship, as shown in figure 7. The abscissa in this figure represents the crack opening displacement, $\delta$, normalized by its critical value, $\delta_{\mathrm{cr}}$, and the ordinate represents the uniform far-field stress, $\sigma$, normalized by the quasistatic fracture strength of the material, $\sigma_{\mathrm{fr}}$. Thus, we could now disregard the presence of microcracks, but instead assume the presence of a fictitious material obeying this cohesive law.

The full line in figure 7 refers to an approximate form of the cohesion law. A typical loading-unloading cycle, oabo, is also shown. When loaded, the solid deforms elastically along path oa, until the full line is reached. This deformation is reversible; if unloaded at this point the material will traverse path $a o$ to reach the original state. Loading beyond point $a$ causes the microcracks to grow irreversibly, along $a b$, until either the material is unloaded (path $b o$ ) or the microcracks coalesce, $\delta=\delta_{\mathrm{cr}}$. For the next loading cycle, the material deforms elastically along path $o b$ up to point $b$. It should be noted that each loading cycle beyond the full line results in lowering of the elastic modulus and the maximum stress the microcracks can withstand, which are essential features of any brittle damage. Hence, as mentioned in the introduction, the cohesive law is equivalent to a constitutive law for the microcracked material, applicable under static or quasistatic loading situations. 


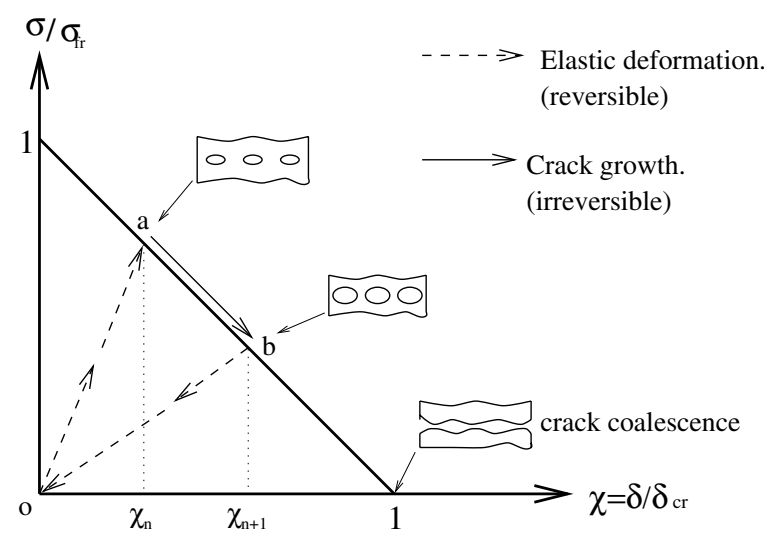

Figure 7. Cohesive law: $\sigma-\delta$ relation.

\section{Model of spall failure}

Acoustic impedance of urinary stones relative to water, $\left((\rho c)_{\text {stone }} /(\rho c)_{\text {water }}\right)$, is found to range between 2 and 5 . The lower value of the above range holds for a composite and highly porous stone material, and the higher value for a uniform, dense composition. There could be several situations in which a tensile stress can be induced in the stone interior, due to an impedance mismatch:

(a) Tensile stress due to reflection of the compressive wavefront from the distal surface of the stone. This is commonly known as the Hopkinson effect, shown in figure 8(a), and is readily observable in stone phantoms. Failure stress first occurs near the reflecting surface, when the wave is reflecting through itself. Tensile stress induced due to this effect will be higher than in the cases listed below, but its effect will be localized near the distal stone surface.

(b) In the first case, we assumed the wave to reflect from a planar boundary, parallel to the shock front. However, a more common situation would be a stone with its boundaries oriented at an angle to the shock front. Figure $8(b)$ shows a shock wave approaching a corner, with the wave front perpendicular to the corner angle bisector. The interaction of the two tensile waves reflected from the sides of the corner induces a high tensile stress at the angle bisector. A detailed calculation of stresses induced for various corner angles and shock front orientations have been reported in literature (Rinehart 1975). Note that in this case the fracture plane is perpendicular to the wave front.

(c) Voids and inhomogeneities present inside the stone also give rise to a tensile reflection. These tensile reflections are localized to a region near the void, and decay rapidly.

(d) A tensile stress is also induced due to the negative pressure in the trailing part of the pulse. The stress induced due to this negative pressure will be lower than the reflected tensile waves discussed in case (a), but it will be effective in the entire stone and not localized to any region. Hence, in subsequent analysis, representative values of the lithotripter negative pressure pulse will be used. Interaction between this trailing pulse and the reflected tensile wave adds to the effect.

Ideally our goal is to predict the size and number of fragments, given a distribution of flaws in an otherwise solid material, subjected to one or more of the above stress scenarios. Figure $9(a)$ is a detailed material model. The springs shown in this figure represent the 
a) Hopkinson effect.

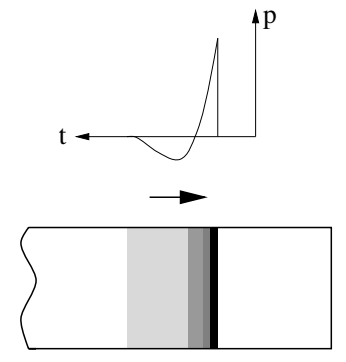

Incident pulse.

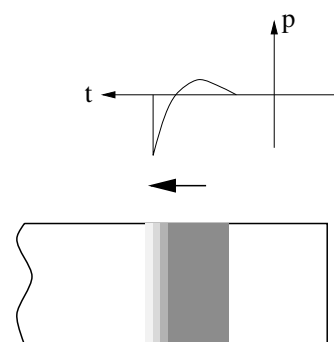

Reflected pulse

b) Reflection from a corner.

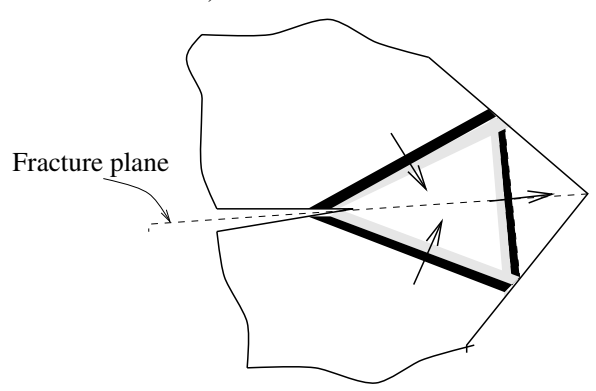

Figure 8. Spall failure: (a) Hopkinson effect, $(b)$ reflection from a corner.

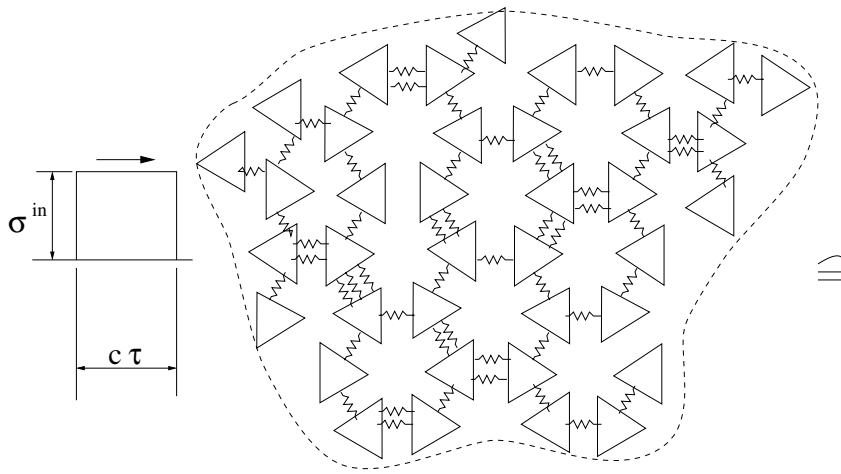

a) $2 \mathrm{D}$ - $\mathrm{n}$ fragments.

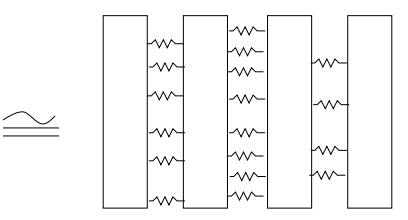

b) $1 \mathrm{~d}-\mathrm{n}$ fragments.

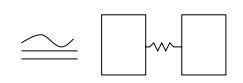

c) 1 d - 2 fragments.

Figure 9. Material models_-varying degrees of complexity.

interlamellar flawed region and they obey the cohesive law, deduced earlier (figure 7). The triangles represent the relatively flaw-free crystalline material. A void in the material is 


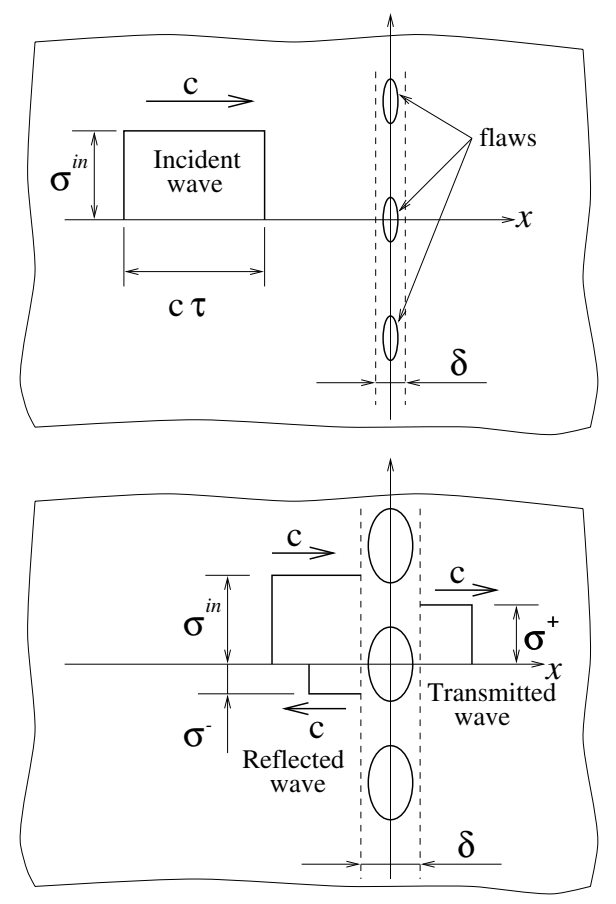

Figure 10. Spall failure model.

represented by an absent spring. This detailed model would allow us to compute the fragment size distribution as a function of shock wave parameters and the number of shock waves. However, calculations for this 2D model (figure $9(a)$ ) would be computationally intensive. A simpler version is the 1D model shown in figure $9(b)$, which would still allow $n$ linear fragments to form, and will be pursued later. In the current work we will consider the simplest case, depicted in figure $9(c)$. It is a one-dimensional model, in which the stone fragments into just two pieces. Physically this model represents a collinear array of uniformly spaced cracks, subjected to a tensile stress pulse of constant magnitude $\sigma^{\text {in }}$ and duration $\tau$ (Camacho and Ortiz 1996), as shown in figure 10. The tensile pulse could arise from either of the scenarios discussed before, hence $\left(\sigma^{\text {in }}, \tau\right)$ are a measure of the tensile portion of the lithotripter pressure pulse. The hypothesized spall plane is located at $x=0$, and the stress pulse is incident on it from the left. This collinear array of cracks obeys the cohesive law. Hence, if the tensile stress exceeds a certain value, as determined by the cohesive law, it will cause the cracks to open up beyond the pre-pulse width of $\delta_{n}$. The objective of this analysis is to obtain a relation between pre-pulse $\left(\delta_{n}\right)$ and post-pulse $\left(\delta_{n+1}\right)$ crack opening, for given values of pulse amplitude and duration $\left(\sigma^{\text {in }}, \tau\right)$, and stone physical properties. Figure 10 shows the interaction between the wave and the array of cracks. The analysis for this configuration is identical to that of wave-interface interaction theory, except that here the interface is of finite width and has a constitutive relation of its own. Acoustics theory relates the stress amplitudes $\sigma$ and the particle velocities $v$ of the incident, reflected and transmitted waves as

$$
\begin{array}{ll}
\sigma^{\text {in }}=-\rho c v^{\text {in }} & \text { incident wave } \\
\sigma^{-}=\rho c v^{-} & \text {reflected wave } \\
\sigma^{+}=-\rho c v^{+} & \text {transmitted wave }
\end{array}
$$


where $\rho$ is the material density and $c$ is the bar-wave speed. Assuming the interface to be of zero mass, force and mass balance at the interface gives

$$
\begin{aligned}
& \sigma^{+}=\sigma^{\text {in }}+\sigma^{-} \\
& \dot{\delta}=v^{+}-\left(v^{\text {in }}+v^{-}\right) .
\end{aligned}
$$

Using (4) to (8) we obtain

$$
\dot{\delta}=\frac{2}{\rho c}\left(\sigma^{\text {in }}(t)-\sigma^{+}(\delta)\right) \quad \text { for } 0 \leqslant t \leqslant \tau .
$$

The constitutive relation for the crack interface is the cohesive law, as depicted in figure 7

$$
\sigma^{+}=\sigma_{\mathrm{fr}}\left(1-\frac{\delta}{\delta_{\mathrm{cr}}}\right)
$$

where the quantities $\delta_{\mathrm{cr}}$ and $\sigma_{\mathrm{fr}}$ have been defined in section 2.2. Substituting (10) in (9) gives

$$
\dot{\delta}-\frac{\delta(t)}{t_{c}}=\frac{2}{\rho c}\left(\sigma^{\mathrm{in}}(t)-\sigma_{\mathrm{fr}}\right)
$$

where $t_{c}=\rho c \delta_{\mathrm{cr}} / 2 \sigma_{\mathrm{fr}}$ provides a characteristic time scale for the process of crack growth. Equation (11) determines the evolution of crack opening $\delta$ with time. Normalizing time ( $t$ and $\tau$ ) by $t_{c}, \delta$ by $\delta_{\mathrm{cr}}, \sigma$ by $\sigma_{\mathrm{fr}}$, denoting these normalized variables by primes, i.e. $t^{\prime}=t / t_{c}$, $\delta^{\prime}=\delta / \delta_{\mathrm{cr}}, \sigma^{\prime}=\sigma / \sigma_{\mathrm{fr}}$ and solving the above equation with boundary conditions: $\delta^{\prime}(0)=$ $\delta_{n}^{\prime}, \delta^{\prime}\left(\tau^{\prime}\right)=\delta_{n+1}^{\prime}$, gives

$$
\delta_{n+1}^{\prime}=\delta_{n}^{\prime} \mathrm{e}^{\tau^{\prime}}+\mathrm{e}^{\tau^{\prime}} \int_{0}^{\tau^{\prime}} \mathrm{e}^{-t^{\prime}}\left(\sigma^{\prime \text { in }}\left(t^{\prime}\right)-1\right) \mathrm{d} t^{\prime} .
$$

For brevity, the primes over the normalized variables are dropped. Equation (12) can be written in the form of a recursive equation

$$
\delta_{n+1}=A \delta_{n}+B
$$

with $\delta_{1}=0$ and $A=\mathrm{e}^{\tau}$. For a stress pulse of constant amplitude, $\sigma^{\text {in }}, B=\left(\sigma^{\text {in }}-1\right)\left(\mathrm{e}^{\tau}-1\right)$. From (13) the crack opening after the $n$th pulse is obtained as

$$
\delta_{n+1}=\frac{A^{n}-1}{A-1} B .
$$

Spalling is said to have occurred when $\delta=\delta_{\mathrm{cr}}$, or in normalized variables $\delta_{N+1}=1$. Solving for $N$, the number of shock waves to spall, in dimensional variables

$$
N=\frac{t_{c}}{\tau} \ln \frac{\sigma^{\text {in }}}{\sigma^{\text {in }}-\sigma_{\mathrm{fr}}} .
$$

As explained earlier in this section, the number of shocks, $N$, calculated using the simplified model (figure $9(c)$ ), will at most fragment the stone into two pieces. In order to calculate the total number of shocks, for generating $n$ fragments, we need to either resort to the detailed model or provide an estimate by assuming some generic features of the fragmentation process. For instance, if the fragmentation process is linear, i.e. a fragment forms after every $N$ shocks, then the the total number of shocks will be $n N$. This would be the worst case scenario. The best case will be a logarithmic fragmentation process, i.e. the number of fragments double after every $N$ shocks, giving a total of $N \log _{2}(n)$ shocks for generating $n$ fragments. The actual fragmentation process might be much more complicated than the above two limiting processes and lack of experimental results on these details only leaves us with the above limits. 


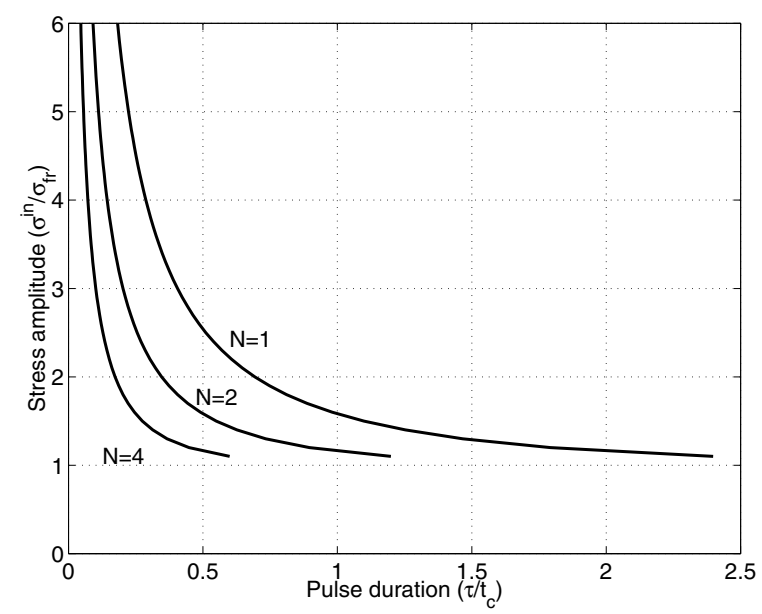

Figure 11. Stress $\left(\sigma^{\text {in }} / \sigma_{\text {fr }}\right)$ versus pulse duration $\left(\tau / t_{c}\right)$ to cause failure, for $N$ pulses.

In the above analysis, material properties on both the sides of the interface were assumed identical. In case of dissimilar materials (we denote their properties by subscripts 1 and 2) one could trace the steps given above, leading to:

$$
N=\frac{t_{c}}{\tau} \ln \frac{T \sigma^{\text {in }}}{T \sigma^{\text {in }}-\sigma_{\text {fr }}}
$$

where $t_{c}=T(\rho c)_{1} \delta_{\mathrm{cr}} / 2 \sigma_{\mathrm{fr}}, T=2 r /(r+1)$ is transmissivity and $r=(\rho c)_{2} /(\rho c)_{1}$ is the ratio of acoustic impedance across the interface. For $r=1,(16)$ becomes identical to (15). Stones having a composite crystalline structure, like layers of calcium monohydrate and uric acid, will have a lower value of $\sigma_{\mathrm{fr}}$ at the interface, and could have $T>1$, indicating a higher susceptibility to fracture at the intercrystalline interface, as opposed to a uniform dense stone.

Figure 11 plots the variation of the pulse duration versus incident stress, with number of shock waves to failure as a parameter. For a fixed $N$, it could be seen that short pulse durations require higher incident stress values for failure and vice versa. A quasi-steady uniaxial tension test predicts a static fracture strength, $\sigma_{\mathrm{fr}}$, only, for all materials, including renal calculi. The variation seen in figure 11 is a clear indicator that this static fracture strength alone does not characterize the process of fracture, which is a dynamic process under lithotripsy conditions.

The assumptions implicit in the above analysis and the limitations imposed by the same, are now discussed:

(a) The cracked region has been treated as an interface, so far as wave propagation is concerned. This implies that $\delta_{\mathrm{cr}} \ll c \tau$, i.e. the crack opening is significantly smaller than the spatial extent of the stress pulse. This is well justified since we are dealing with intergranular cracks of the order of $1 \mu \mathrm{m}$ wide, whereas the lithotripter pulse lasts for $4 \mu \mathrm{s}$ (i.e. $c \tau \sim 2000 \times 4 \times 10^{-6} \sim 1 \mathrm{~cm}$ wide). For all voids and inhomogeneities, inside the stone, which have a dimension several orders smaller than the above length scale $(1 \mathrm{~cm})$, this analysis holds. For larger flaws, a more detailed wave-crack interaction theory would be needed.

(b) The cohesive law discussed in section 2.2 is applicable for quasistatic crack growth, i.e. the $\sigma-\delta$ relation assumed in this analysis holds for a very slow crack growth speed. Now, equation (15) gives the total time for spalling to occur, i.e. the total time taken for a flaw of 


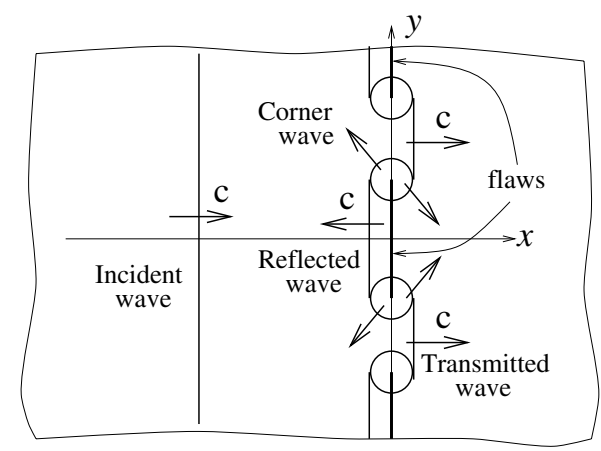

Figure 12. Detailed wave-crack interaction.

length $a_{0}$ to grow to a length $l$. Thus, this theory predicts an apparent crack propagation speed, $\overline{\dot{a}}$, which is calculated as follows:

$$
\overline{\dot{a}}=\frac{\left(l-a_{0}\right) / 2}{N \tau} \approx \frac{l / 2}{t_{c} \ln \left(\sigma^{\mathrm{in}} /\left(\sigma^{\mathrm{in}}-\sigma_{\mathrm{fr}}\right)\right)}
$$

where we have assumed $a_{0} \ll l$. Substituting for $t_{c}, \sigma_{\mathrm{fr}}, \delta_{\mathrm{cr}}$, and normalizing $\overline{\dot{a}}$ by bar-wave speed, $c,(17)$ takes the form

$$
\overline{\dot{a}}=\frac{\pi}{8} \frac{1}{1-v^{2}}\left(\frac{2}{\pi} \tan \frac{\pi a_{0}}{2 l}\right)^{-1 / 2}\left[\ln \left(\frac{\sigma^{\text {in }}}{\sigma^{\text {in }}-\sigma_{\mathrm{fr}}}\right)\right]^{-1} .
$$

In (18) we also used the relation for the bar speed, $E / \rho c^{2}=1$. Substituting some typical values, like $a_{0} / l=0.2, v=0.3$ and $\sigma^{\text {in }} / \sigma_{\mathrm{fr}}=1.6$, in the above expression, gives $\overline{\dot{a}} \approx 1$. Higher values of $\sigma^{\text {in }} / \sigma_{\text {fr }}$ and lower values of $a_{0} / l$ will result in an apparent crack speed higher than the wave speed, which is not permissible.

(c) Figure 12 depicts the detailed wave-crack interaction. The incident pulse interacts with each crack and sends a reflected and a transmitted wave, with a radial corner wave originating at the crack tips. The radial corner waves expand with time, and eventually reach adjacent cracks. An implication of using the quasistatic model (i.e. cohesive law) for crack growth is that these radial waves have propagated past the adjacent cracks several times and an equilibrium is attained along the interface in the $y$ direction. This requires that the length of the interface (along the $y$-axis in figure 12) should be smaller than the spatial extent of the stress pulse, i.e. $l \times$ number of cracks $\ll c \tau$. This assumption is not true for length scales of the order of the stone size $(1 \mathrm{~cm})$; however, it is true for a small region of the interlamellar crack which could be $100 \mu \mathrm{m}$ long. Thus the number of shock waves calculated, $N$, will only cause a small portion of the stone to lose cohesion rather than fragment it into two pieces.

Despite of the shortcomings of this model it is considered useful, since it predicts the qualitative features of transient microcrack damage accumulation. Several related issues like crack branching, bifurcation, etc, which are of relevance to fragmentation have not been considered, since no mechanistic understanding of these processes are available as yet. Although there are several other models for the same problem (e.g. Fruend 1990), we favour this model primarily due to its simplicity. 
Table 1. Mechanical properties of calcium oxalate monohydrate (COM) calculus.

\begin{tabular}{llll}
\hline Density $(\rho)$ & Acoustic speed $(c)$ & $\delta_{\text {cr }}$ & $\sigma_{\text {fr }}$ \\
\hline $2550 \mathrm{~kg} \mathrm{~m}^{-3}$ & $4500 \mathrm{~m} \mathrm{~s}^{-1} \dagger$ & $5 \mu \mathrm{m}$ & $1 \mathrm{MPa}$, in tension $\neq$ \\
\hline
\end{tabular}

$\uparrow$ Chuong et al (1993).

$\$$ Cohen (1993).

\section{Number of shock waves to failure}

We now calculate the number of shock waves for a spall-like failure to occur, based on the theory formulated in section 3. Typically, a calculus having a characteristic dimension of $1 \mathrm{~cm}$ is broken into fragments of dimension $1 \mathrm{~mm}$ or smaller, which amounts to 1000 fragments. The number of shock waves administered to achieve this fragment size distribution ranges from 1000 to 3000 . The mechanical properties of calcium oxalate monohydrate (COM) calculus are given in table 1 . The value of $\delta_{\mathrm{cr}}$ is related to fracture toughness (equation (3)); since fracture toughness of $\mathrm{COM}$ has not been measured yet the value of $\delta_{\mathrm{cr}}$ tabulated above is a rough estimate (using $K_{I C} \approx 10 \mathrm{MPa} \mathrm{m}^{1 / 2}, v=0.3, l \approx 10 \mu \mathrm{m}, E \approx 1 \mathrm{GPa}$ ). Based on the above values, the characteristic time scale, $t_{c}$, is estimated as $28 \mu \mathrm{s}$. As mentioned earlier, free-field measurements near the lithotripter focus indicate a negative pressure of $10 \mathrm{MPa}$, for a time span of $2 \mu \mathrm{s}$. Assuming a 50\% attenuation of this pulse during in vivo fragmentation, (15) gives

$$
N=\frac{t_{c}}{\tau} \ln \frac{\sigma^{\text {in }}}{\sigma^{\text {in }}-\sigma_{\mathrm{fr}}}=\frac{28}{2} \ln \frac{5}{5-1} \approx 3 .
$$

As explained before, if $n$ fragments are desired, then the total number of shocks required in the course of treatment can lie between a maximum of $n N=3000$, to a minimum of $\log _{2}(n) N=30$, depending on how the process of fragmentation takes place. In the actual treatment the total number of shocks will be somewhere in between the above limits.

\section{Tissue injury versus stone comminution}

As mentioned in the introduction, we demonstrate here a means of manipulating shock wave parameters which will minimize tissue injury and enhance stone comminution by exploiting the differences in the way these progressive processes develop. A quantitative description of damage to stone follows logically from the theory presented in section 3 . For a planar array of collinear cracks, the ratio $a / l$ could be a definition of damage. Since there is a unique relation between $a / l$ and $\delta / \delta_{\text {cr }}$, the latter is also a good candidate for describing damage. Thus, we define a damage variable, $\chi$, as

$$
\chi=\delta / \delta_{\mathrm{cr}} \text {. }
$$

$\chi=0$ corresponds to absence of cracking and $\chi=1$ corresponds to a fully cracked situation. Using the above definition in (13), we have an equation for damage evolution

$$
\chi_{n, \text { sto }}=A_{n, \text { sto }} \chi_{n-1, \text { sto }}+B_{n, \text { sto }}
$$

here the subscripts $n$, sto indicate the number of shocks and stone, respectively. In (21), $A$ and $B$ are subscripted by $n$ to allow for a very general loading situation, in which $\left(\sigma_{\text {in }}, \tau\right)$ could be different for each pulse.

In shock-wave lithotripsy (SWL) tissue injury is concomitant with stone comminution. Experiments on thin planar polymeric membranes (Howard and Sturtevant 1997) suggested 'shearing' as the damage mechanism. This shearing effect was attributed to the gradient 


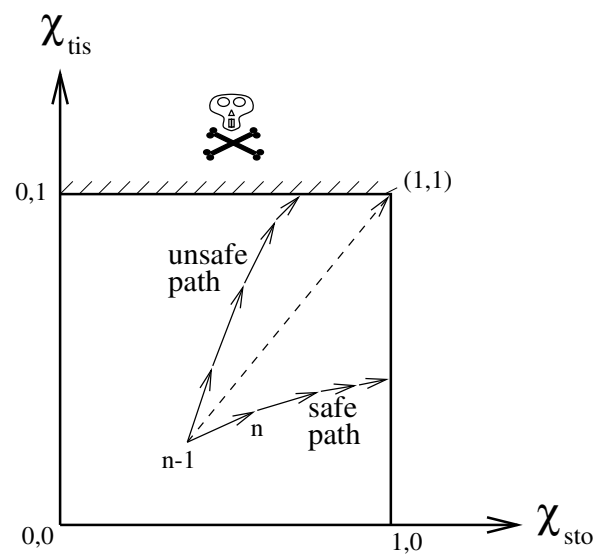

Figure 13. Evolution of $\left(\chi_{\text {sto }}, \chi_{\text {tis }}\right)$ with successive pulses.

in shock strength along the shock front. Experiments on aluminium foil (Bailey et al 1999) indicate pitting damage, implicating cavitation as the damage mechanism. We again emphasize here that although the fluid flow-field for the above two mechanisms (bubble implosion or gradient in shock strength) is different, the damage process in the tissue and stone material is identical, i.e. dynamic fatigue. The postulate used for describing damage to stone-presence of micro-flaws which grow, coalesce and eventually pervade the entire material-is applicable to tissue material as well. Thus both the tissue damage mechanisms can be described by a damage evolution relation of the form (21), albeit the parameters $A$ and $B$ will have a different definition for each mechanism. The definition of $A$ and $B$ will also depend on material constitution, namely tissue and calculi.

Suppose tissue damage evolves as

$$
\chi_{n, \text { tis }}=A_{n, \text { tis }} \chi_{n-1, \text { tis }}+B_{n, \text { tis }}
$$

where the subscript 'tis' indicates tissue in the above equation. The $A \mathrm{~s}$ and $B \mathrm{~s}$ used in the above equation depend on tissue properties and $\left(\sigma^{\text {in }}, \tau\right)$, and are hence different from that of the stone. The evolution of damage during SWL can be described on a $\left(\chi_{\text {sto }}, \chi_{\text {tis }}\right)$ plot. Initially, the stone and the tissue are in an undamaged state $\left(\chi_{\text {sto }}, \chi_{\text {tis }}\right)=(0,0)$. With subsequent application of shock waves, damage evolves according to (21) and (22), and traces a path on the $\left(\chi_{\text {sto }}, \chi_{\text {tis }}\right)$ plot, as shown in figure 13. The square box formed by the two axes, and the lines $\chi_{\text {sto }}=1$ and $\chi_{\text {tis }}=1$ bounds the damage evolution process. A desirable damage evolution is one in which $\chi_{\text {sto }}=1$ is reached, representing a successful completion of treatment, with $\chi_{\text {tis }}<1$. This may be called as a 'safe path'. Contrary to this safe path is an 'unsafe path', in which $\chi_{\text {tis }}=1$ is reached but $\chi_{\text {sto }}<1$. The broken line shown in figure 13 demarcates a safe from an unsafe damage evolution. Hence, at any instant, if the damage evolution has to be safe then the following restriction needs to be imposed:

$$
\frac{\chi_{n, \text { tis }}-\chi_{n-1, \text { tis }}}{\chi_{n, \text { sto }}-\chi_{n-1, \text { sto }}}<\frac{\chi_{n-1, \text { tis }}-1}{\chi_{n-1, \text { sto }}-1} .
$$

Substituting (21) and (22) in (23) gives

$$
\frac{\left(A_{n, \text { tis }}-1\right) \chi_{n-1, \text { tis }}+B_{n, \text { tis }}}{\left(A_{n, \text { sto }}-1\right) \chi_{n-1, \text { sto }}+B_{n, \text { sto }}}<\frac{\chi_{n-1, \text { tis }}-1}{\chi_{n-1, \text { sto }}-1} .
$$


Solving (24), yields combinations of $\left(\sigma^{\text {in }}, \tau\right)$ for some safe damage evolution

$$
\frac{\sigma^{\text {in }}}{\sigma_{\text {fr,sto }}}<\frac{\left(\chi_{n-1, \text { sto }}-1\right)\left(\frac{\mathrm{e}^{\tau / t_{c} \text { sto }}-\mathrm{e}^{\tau / t_{c, \text { tis }}}}{\mathrm{e}^{\tau / t_{c} \text { sto }}-1}\right)}{\frac{\sigma_{\text {fr, sto }}}{\sigma_{\text {fr, tis }}}\left(\frac{\mathrm{e}^{\tau / t_{c, \text { tis }}}-1}{\mathrm{e}^{\tau / t_{c, \text { sto }}}-1}\right)\left(\frac{\chi_{n-1, \text { sto }}-1}{\chi_{n-1, \text { tis }}-1}\right)-1} .
$$

Thus, as seen in (25), a safe combination of incident stress pulse $\left(\sigma^{\text {in }}\right)$ and pulse duration $(\tau)$ depends on stone and tissue fracture properties, $\left(\sigma_{\mathrm{fr}}, t_{c}\right)$, and their damage state, $\left(\chi_{\mathrm{sto}}, \chi_{\mathrm{tis}}\right)$. Other constraints could be imposed on the damage evolution path, for example given a fixed number of pulses, $N$, minimize $\chi_{N, \text { tis }}$ and attain $\chi_{N \text {,sto }}=1$.

\section{Discussion}

Fatigue damage to kidney stones and tissue material, due to ESWL shock waves, has been analysed in this work. Experimental observations of the fracture behaviour of brittle materials have indicated a typical dependence of failure stress and the resulting fragment size on strain rate. Here we applied a damage model to analyse spall failure of stone. Primary results from this analysis are discussed below:

(a) The number of shock waves for spall failure were calculated, in terms of shock wave parameters (pulse amplitude and duration) and material properties. The number calculated here $(N)$, has a range of two orders of magnitude (30-3000). This is attributed to:

- As discussed earlier, the model used in this calculation is an over-simplification of the material characteristics and thus has limitations in describing the fragmentation process. The fragment size distribution of the crushed stone governs the number of shock waves; however, the model used here does not incorporate this dependence. A more detailed model, as explained in figure 9, with a more realistic flaw distribution (Weibull 1951), instead of the assumed regular flaw distribution, would remedy the situation and constitutes our future work. Also, experimental results providing the details of the fragmentation process, in vivo, are lacking, and are needed to help us improve this model.

- Mechanisms other than spall are also significant in inflicting damage to stone and tissue, and spall may be operative in a small portion of the stone. In the introduction a mechanism was explained whereby an overall compressive stress induces local tensile stresses, causing microcracks to grow. In subsequent work we intend to develop this model and perform a similar calculation for number of shock waves for failure.

- Experimental data about the fracture properties, e.g. fracture toughness, of renal calculi are unavailable. The models used here rely heavily on such data.

Besides predicting the number of shock waves for an actual lithotripsy treatment, the utility of this calculation is also as an expression relating the various stone and shock wave parameters. The notion of a relative fragility parameter, based on the number of shock waves required to attain a given fragment size distribution, has been quite common amongst earlier investigators. The relation of this parameter with various stone properties has been studied (see the review paper by Coleman and Saunders (1993)). The analysis presented in this work provides a relation between this relative fragility parameter and the stone properties, for given shock wave parameters. Let $f$ denote the relative fragility 
parameter, then from (16), $f$ is evaluated as

$$
f=\frac{1 / N_{\text {stone }}}{1 / N_{\text {ref }}}=\frac{\left(\frac{t_{c}}{\tau} \ln \frac{T \sigma^{\text {in }}}{\left(T \sigma^{\text {in }}-\sigma_{\mathrm{fr}}\right)}\right)_{\text {ref }}}{\left(\frac{t_{c}}{\tau} \ln \frac{T \sigma^{\text {in }}}{\left(T \sigma^{\text {in }}-\sigma_{\mathrm{fr}}\right)}\right)_{\text {stone }}} .
$$

Thus $f$ denotes the ease with which a stone fragments relative to a reference phantom or other stone.

(b) The process of SWL was described in terms of damage evolution of tissue and stone material. Equation (25) suggests a way of manipulating the shock wave parameters, as the treatment progresses, which would allow us to control tissue and stone damage. In developing this damage evolution model, the definition of damage to stone, i.e. impairment of mechanical properties, was extended to tissue material as well. Impairment of the mechanical properties of tissue, during SWL, is due to destruction at a cellular level. A more refined description of tissue damage would account for this cellular level damage; for instance, the fraction of cells lysed with each shock pulse. Relating this fraction with shock wave parameters would enable us to build a better damage evolution model, and increase treatment efficiency.

\section{Acknowledgments}

We thank Professor G Ravichandran and Professor M Ortiz for their valuable comments on the ideas discussed in this work. This work was supported by NIH grant P01 DK43881.

\section{References}

Achenbach J D, Keer L M, Khetan R P and Chen S H 1979 Loss of adhesion at the tip of an interface J. Elasticity 9 $397-424$

Bailey M R, Blackstock D T, Cleveland R O and Crum L A 1998 Comparison of electrohydraulic lithotripters with rigid and pressure-release ellipsoidal reflectors: I. Acoustic fields J. Acoust. Soc. Am. 104 2517-24

1999 Comparison of electrohydraulic lithotripters with rigid and pressure-release ellipsoidal reflectors: II. Cavitation fields J. Acoust. Soc. Am. 106 1149-60

Barenblatt G I 1962 The mathematical theory of equilibrium cracks in brittle fracture Adv. Appl. Mech. 7 55-129

Blake J R and Gibson D C 1987 Cavitation bubbles near boundaries Ann. Rev. Fluid Mech. 1999-123

Brace W F and Bombolakis E G 1963 A note on brittle crack growth in compression J. Geophys. Res. 68 3709-13

Bombolakis E G 1968 Photoelastic study of initial stages of brittle fracture in compression Tectonophysics 6 461-73

Camacho G T and Ortiz M 1996 Computational modelling of impact damage in brittle materials Int. J. Solids Structures 20 2899-938

Chaussy Ch and Fuchs G J 1989 Current state and future developments of non-invasive treatment of human urinary stones with ESWL J. Endourol. 141 782-9

Chuong C J, Zhong P and Preminger M 1993 Acoustic and mechanical properties of renal calculi: implications in SWL J. Endourol. 7 437-44

Cohen N P and Whitfield H N 1993 Mechanical testing of urinary calculi World J. Urology 11 13-18

Coleman A J and Saunders J E 1993 A review of the physical properties and biological effects of the high amplitude acoustic fields used in extracorporeal lithotripsy Ultrasonics 31 75-89

Coleman A J, Saunders J E, Crum L A and Dyson M 1987 Acoustic cavitation generated by an extracorporeal shockwave lithotripter Ultrasound Med. Biol. 13 69-76

Dugdale D S 1960 Yielding of sheet steel containing slits J. Mech. Phys. Solids 8 100-4

Ebrahimi F and Wang F 1989 Fracture behaviour of urinary stones under compression J. Biomed. Mater. Res. 23 507-21

Fruend L B 1990 Dynamic Fracture Mechanics (Cambridge: Cambridge University Press)

Griffith A A 1921 The phenomenon of rupture and flow in solids Phil. Trans. R. Soc. (London) 22 163-98 
Howard D D and Sturtevant B 1997 In vitro study of the mechanical effects of shock wave lithotripsy Ultrasound Med. Biol. 23 1107-22

Kachanov L M 1986 Introduction to Continuum Damage Mechanics (Boston: Kluwer)

Kaude J V, Williams C M, Millner M R, Scott K N and Finlayson B 1985 Renal morphology and function immediately after extracorporeal shock-wave lithotripsy Am. J. Roentgenol 145 305-13

Miner M A 1945 Cumulative damage in fatigue J. Appl. Mech. A159-A164

Müller M 1990 Comparison of Dornier lithotripters: measurement of shock wave fields and fragmentation effectiveness Biomed. Tech. 35 250-62

Needleman A 1987 A continuum model for void nucleation by inclusion debonding J. Appl. Mech. 54 525-31

Nemat-Nasser S and Deng H 1994 Strain-rate effect on brittle failure in compression Acta Metall. Mater. 42 1013-24

Ortiz M 1988 Microcrack coalescence and macroscopic crack growth initiation in brittle solids Int. J. Solids Structures 24 231-50

Rinehart J 1975 Stress Transients in Solids (Santa Fe, NM: Hyperdynamics)

Robin C, Bailey M, Fineberg N, Hartenbaum B, Lokhandwalla M, McAteer J and Sturtevant B 2000 Design and characterisation of a research electrohydraulic lithotripter patterned after the Dornier HM3 Rev. Sci. Instrum. 71 (6)

Sturtevant B and Kulkarny V A 1976 The focusing of weak shock waves J. Fluid. Mech. 73 651-71

Weibull W J A 1951 Statistical distribution function of wide applicability J. Appl. Mech. 18 293-7 